\title{
A physically-motivated photometric calibration of M dwarf metallicity
}

\author{
K. C. Schlaufman and G. Laughlin
}

\begin{abstract}
Astronomy and Astrophysics Department, University of California, Santa Cruz, CA 95064, USA
e-mail: [kcs, laugh]@ucolick.org
\end{abstract}

Received 18 May 2010 / Accepted 11 June 2010

\begin{abstract}
The location of $\mathrm{M}$ dwarfs in the $\left(V-K_{\mathrm{s}}\right)-M_{K \mathrm{~s}}$ color-magnitude diagram (CMD) has been shown to correlate with metallicity. We demonstrate that previous empirical photometric calibrations of $\mathrm{M}$ dwarf metallicity exploiting this correlation systematically underestimate or overestimate metallicity at the extremes of their range. We improve upon previous calibrations in three ways. First, we use both a volume-limited and kinematically-matched sample of F and G dwarfs from the Geneva-Copehnagen Survey (GCS) to infer the mean metallicity of M dwarfs in the Solar Neighborhood. Second, we use theoretical models of M dwarf interiors and atmospheres to determine the effect of metallicity on M dwarfs in the $\left(V-K_{\mathrm{s}}\right)-M_{K \mathrm{~s}}$ CMD. Third, though we use the GCS to infer the mean metallicity of M dwarfs in the Solar Neighborhood, our final calibration is based purely on high-resolution spectroscopy of FGK primaries with M dwarf companions as well as the trigonometric parallaxes and apparent $V$ - and $K_{\mathrm{s}}$-band magnitudes of those $\mathrm{M}$ dwarf companions. As a result, our photometric calibration explains an order of magnitude more of the variance in the calibration sample than previous photometric calibrations. We use our calibration to non-parametrically quantify the significance of the observation that $\mathrm{M}$ dwarfs that host exoplanets are preferentially in a region of the $\left(V-K_{\mathrm{s}}\right)-M_{K \mathrm{~s}}$ plane populated by metal-rich $\mathrm{M}$ dwarfs. We find that the probability $p$ that planet-hosting $\mathrm{M}$ dwarfs are distributed across the $\left(V-K_{\mathrm{s}}\right)-M_{K \mathrm{~s}} \mathrm{CMD}$ in the same way as field $\mathrm{M}$ dwarfs is $p=0.06 \pm 0.008$. Interestingly, the subsample of $\mathrm{M}$ dwarfs that host Neptune and sub-Neptune mass planets may also be preferentially located in the region of the $\left(V-K_{\mathrm{s}}\right)-M_{K \mathrm{~s}}$ plane populated by high-metallicity $\mathrm{M}$ dwarfs. The probability of this occurrence by chance is $p=0.40 \pm 0.02$, and this observation hints that low-mass planets may be more likely to be found around metal-rich M dwarfs. The confirmation of this hint would be in contrast to the result obtained for FGK stars, where it appears that metal-rich and metal-poor stars hosts Neptune-mass planets with approximately equal probability. An increased rate of low-mass planet occurrence around metal-rich $\mathrm{M}$ dwarfs would be a natural consequence of the core-accretion model of planet formation.
\end{abstract}

Key words. planets and satellites: formation - stars: abundances - stars: low-mass - stars: statistics

\section{Introduction}

The determination of metallicity for $\mathrm{M}$ dwarfs is a very difficult problem (e.g. Gustafsson 1989). Their cool atmospheres permit the existence of many molecules for which molecular opacities are currently poorly constrained. As a result, the estimation of the continuum level of a spectrum is challenging, rendering linebased metallicity indicators unreliable. The poorly constrained molecular opacity data currently available makes the determination of metallicity through spectral synthesis difficult as well. For those reasons, alternative methods must be employed to estimate $\mathrm{M}$ dwarf metallicities.

The main sequence lifetimes of $\mathrm{M}$ dwarfs are longer than the Hubble time, so they have not yet departed much from the zero-age main sequence. Consequently, $M$ dwarfs might be expected to form a two-parameter sequence in mass and metallicity, suggesting that a two-color broad-band photometric calibration might constrain their properties. There have been several attempts to obtain the metallicity of $\mathrm{M}$ dwarfs using their photometric properties, including two recent breakthroughs. Bonfils et al. (2005a) - B05 hereafter - had the subtle insight to realize that $\mathrm{M}$ dwarfs in binary or multiple systems should have metallicities commensurate with the easily-measured metallicity of an FGK primary in the system. In that way, B05 identified a calibration sample of $\mathrm{M}$ dwarfs with metallicities securely determined in one of two ways: (1) high-resolution spectroscopy of an FGK companion and (2) high-resolution spectroscopy of
M dwarfs for which $T_{\text {eff }}$ and $\log g$ could be fixed with photometric data. For the former, the metallicity is very likely the same as the metallicity inferred from high-resolution spectroscopy of its FGK companion. For the latter, spectral synthesis after fixing $T_{\text {eff }}$ and $\log g$ with photometric data eliminates some degeneracy and produces a reasonable metallicity estimate. They noted that low-metallicity $\mathrm{M}$ dwarfs have blue $V-K_{\mathrm{s}}$ color at constant $K_{\mathrm{s}}$-band absolute magnitude $M_{K \mathrm{~s}}$, and they fit a linear model to their calibration sample using $V-K_{\mathrm{s}}$ and $M_{K \mathrm{~s}}$ to predict $[\mathrm{Fe} / \mathrm{H}]$. Johnson \& Apps (2009) - JA09 hereafter - addressed the relative lack of high-metallicity $\mathrm{M}$ dwarfs in the calibration sample of B05 and created an empirical model in which the distance of an $\mathrm{M}$ dwarf above the field $\mathrm{M}$ dwarf main sequence (MS) in the $\left(V-K_{\mathrm{s}}\right)-M_{K \mathrm{~s}}$ color-magnitude diagram (CMD) indicated its metallicity. The great insight of JA09 was that the mean metallicity of a population of $\mathrm{M}$ dwarfs could be characterized by the easily-measured mean metallicity of a similar population of FGK stars. Indeed, they assumed that the field M dwarf MS was an isometallicity contour with the same metallicity as a volumelimited sample of $\mathrm{G}$ and $\mathrm{K}$ stars and fit a linear model using the distance above the field $\mathrm{M}$ dwarf $\mathrm{MS}$ to predict $[\mathrm{Fe} / \mathrm{H}]$.

M dwarfs are attractive targets around which to search for low-mass planets because they have large reflex velocities and transit depths even for low-mass and small-radius companions. Given that the metallicity of protoplanetary disks is a key parameter in models of planet formation (e.g. Laughlin et al. 2004; 
A\&A 519, A105 (2010)

Table 1. M dwarfs in binary systems with an FGK primary and those that host planets.

\begin{tabular}{|c|c|c|c|c|c|c|c|c|c|}
\hline System & $V$ & $\begin{array}{c}K_{\mathrm{s}} \\
{[\mathrm{mag}]}\end{array}$ & $\begin{array}{c}\pi \\
{[\mathrm{mas}]}\end{array}$ & $\begin{array}{l}V-K_{\mathrm{s}} \\
{[\mathrm{mag}]}\end{array}$ & $\begin{array}{c}M_{K \mathrm{~s}} \\
{[\mathrm{mag}]}\end{array}$ & $\begin{array}{c}\text { Spectroscopic } \\
{[\mathrm{Fe} / \mathrm{H}]}\end{array}$ & $\begin{array}{c}\text { Photometric } \\
{[\mathrm{Fe} / \mathrm{H}]}\end{array}$ & Comment & Reference \\
\hline Gl 105B & 11.67 & 6.57 & 138.72 & 5.1 & 7.28 & -0.19 & -0.14 & $\cdots$ & $1,2,3,4$ \\
\hline G1 107B & 10.06 & 5.87 & 89.03 & 4.19 & 5.62 & -0.03 & 0.01 & $\cdots$ & $1,2,4,5$ \\
\hline Gl 166C & 11.17 & 5.9 & 198.08 & 5.27 & 7.38 & -0.33 & -0.05 & $\cdots$ & $1,2,4,5$ \\
\hline Gl 212 & 9.8 & 5.76 & 80.13 & 4.04 & 5.28 & 0.04 & 0.09 & $\ldots$ & $1,2,4,6$ \\
\hline Gl 231.1B & 13.42 & 8.28 & 51.76 & 5.14 & 6.85 & -0.02 & 0.12 & $\ldots$ & $1,2,4,5$ \\
\hline Gl 250B & 10.09 & 5.72 & 114.94 & 4.37 & 6.02 & -0.15 & -0.07 & $\ldots$ & $1,2,4,5$ \\
\hline Gl 297.2B & 11.8 & 7.42 & 44.47 & 4.38 & 5.66 & -0.09 & 0.13 & $\cdots$ & $1,2,3,4$ \\
\hline Gl 324B & 13.14 & 7.67 & 76.8 & 5.47 & 7.10 & 0.32 & 0.26 & $\cdots$ & $1,2,4,5$ \\
\hline Gl 53.1B & 13.6 & 8.67 & 48.2 & 4.93 & 7.09 & 0.07 & -0.17 & $\cdots$ & $1,2,4,5$ \\
\hline Gl 768.1B & 13.1 & 8.01 & 51.57 & 5.09 & 6.57 & 0.07 & 0.22 & $\cdots$ & $1,2,4,5$ \\
\hline Gl 783.2B & 13.94 & 8.88 & 48.83 & 5.06 & 7.32 & -0.16 & -0.19 & $\cdots$ & $1,2,4,5$ \\
\hline Gl 797B & 11.88 & 7.42 & 47.65 & 4.46 & 5.81 & -0.07 & 0.11 & $\cdots$ & $1,2,4,5$ \\
\hline Gl 81.1B & 11.21 & 7.6 & 29.43 & 3.61 & 4.94 & 0.09 & -0.08 & $\cdots$ & $1,2,4,5$ \\
\hline HD 46375B & 11.8 & 7.84 & 29.93 & 3.96 & 5.13 & 0.24 & 0.12 & $\cdots$ & $2,4,7$ \\
\hline HD 38529B & 13.35 & 8.8 & 23.57 & 4.55 & 5.66 & 0.45 & 0.27 & $\cdots$ & $2,4,7$ \\
\hline HD $18143 C$ & 13.86 & 8.66 & 43.71 & 5.2 & 6.84 & 0.28 & 0.17 & $\cdots$ & $2,4,7$ \\
\hline 55 Cnc B & 13.15 & 7.67 & 79.8 & 5.48 & 7.21 & 0.31 & 0.20 & $\cdots$ & $2,4,7$ \\
\hline HD 190360B & 14.4 & 8.71 & 62.92 & 5.69 & 7.71 & 0.21 & 0.10 & $\cdots$ & $2,4,7$ \\
\hline Proxima Cen & 11.11 & 4.38 & 772.33 & 6.73 & 8.81 & 0.21 & 0.14 & $\ldots$ & $2,4,7$ \\
\hline HIP 79431 & 11.34 & 6.589 & 69.46 & 4.751 & 5.80 & $\cdots$ & 0.35 & Jupiter & $2,8,9$ \\
\hline GJ 876 & 10.16 & 5.01 & 213.28 & 5.15 & 6.65 & $\cdots$ & 0.23 & 2 Jupiters + Super-Earth & $2,8,10$ \\
\hline GJ 317 & 12 & 7.028 & 109 & 4.972 & 7.22 & $\cdots$ & -0.20 & Jupiter & 2,11 \\
\hline GJ 849 & 10.42 & 5.594 & 109.94 & 4.826 & 5.80 & $\cdots$ & 0.41 & Jupiter & $2,8,12$ \\
\hline GJ 179 & 11.96 & 6.942 & 81.38 & 5.018 & 6.49 & $\cdots$ & 0.20 & Jupiter & $2,8,13$ \\
\hline GJ 832 & 8.66 & 4.501 & 201.87 & 4.159 & 6.03 & $\cdots$ & -0.24 & Jupiter & $2,8,14$ \\
\hline GJ 649 & 9.7165 & 5.624 & 96.67 & 4.0925 & 5.55 & $\cdots$ & -0.03 & Saturn & $2,8,15$ \\
\hline GJ 436 & 10.67 & 6.073 & 98.61 & 4.597 & 6.04 & $\cdots$ & 0.10 & Neptune & $2,8,16$ \\
\hline GJ 581 & 10.57 & 5.836 & 160.91 & 4.734 & 6.87 & $\cdots$ & -0.22 & Neptune +3 Super-Earths & $2,8,17$ \\
\hline GJ 674 & 9.36 & 4.855 & 220.24 & 4.505 & 6.57 & $\cdots$ & -0.24 & Neptune & $2,8,18$ \\
\hline GJ 176 & 9.97 & 5.607 & 107.83 & 4.363 & 5.77 & $\cdots$ & 0.06 & Neptune & $2,8,19$ \\
\hline GJ 1214 & 14.71 & 8.782 & 77.2 & 5.928 & 8.22 & $\cdots$ & 0.28 & Super-Earth & $2,20,21$ \\
\hline
\end{tabular}

References. (1) Bonfils et al. (2005a); (2) Skrutskie et al. (2006); (3) Mermilliod et al. (1997); (4) Perryman et al. (1997); (5) Gliese \& Jahreiß (1991); (6) Egret et al. (1992); (7) Johnson \& Apps (2009); (8) van Leeuwen (2007); (9) Apps et al. (2010); (10) Marcy et al. (1998); (11) Johnson et al. (2007); (12) Butler et al. (2006); (13) Howard et al. (2010); (14) Bailey et al. (2009); (15) Johnson et al. (2010); (16) Maness et al. (2007); (17) Bonfils et al. (2005b); (18) Bonfils et al. (2007); (19) Forveille et al. (2009); (20) Charbonneau et al. (2009); (21) J. Irwin et al. (priv. comm.).

Ida \& Lin 2004), the metallicity of M dwarfs that host planets will constrain the planet formation process in low-mass protoplanetary disks. Indeed, it is well-established that metal-rich FGK stars are more likely to host giant planets (e.g. Santos et al. 2004; Fischer \& Valenti 2005), but there is also evidence to suggest that metal-rich FGK stars are not much more likely to host Neptune-mass planets than their low-metallicity counterparts (e.g. Udry et al. 2006; Sousa et al. 2008; Bouchy et al. 2009). Already, JA09 have used their model of M dwarf metallicity to suggest that the M dwarfs that host planets are preferentially metal-rich. However, JA09 did not address whether the apparent lack of a correlation between FGK host stellar metallicity and the presence of Neptune-mass planets extends to $\mathrm{M}$ dwarfs.

In this paper, we examine a calibration sample of $\mathrm{M}$ dwarfs with securely estimated metallicities and we show that the models of B05 and JA09 systematically underestimate or overestimate metallicity at the extremes of the range of this calibration sample. We demonstrate that a volume-limited and kinematically-matched sample of Sun-like stars produces a better estimate of the mean $M$ dwarf metallicity in the Solar Neighborhood, and we use M dwarf models of different metallicities from Baraffe et al. (1998) to improve on the technique described in JA09. The position of an M dwarf in the $\left(V-K_{\mathrm{S}}\right)-$ $M_{K \mathrm{~s}} \mathrm{CMD}$ remains an indicator of its metallicity, and we use that fact to non-parametrically quantify the significance of the observation that planet-hosting $\mathrm{M}$ dwarfs are preferentially in a region of the $\left(V-K_{\mathrm{s}}\right)-M_{K \mathrm{~s}}$ plane populated by metal-rich $\mathrm{M}$ dwarfs. Moreover, we identify for the first time a hint that the subsample of $\mathrm{M}$ dwarfs that host Neptune and sub-Neptune mass planets may also be more likely to be in the region of the $\left(V-K_{\mathrm{s}}\right)-M_{K \mathrm{~s}} \mathrm{CMD}$ associated with metal-rich M dwarfs. We describe our analysis in Sect. 2 and summarize our findings in Sect. 3.

\section{Analysis}

\subsection{Testing previous calibrations}

We first collect from B05 and JA09 a calibration sample of M dwarfs in wide binary or multiple systems with an FGK primary. The metallicity of the FGK primary in the system is straightforward to measure from a high-resolution spectrum, and if the $\mathrm{M}$ dwarf secondary and the FGK primary formed in the same molecular core, then the expectation is that the two should have commensurate metallicities. We collect 13 examples from $\mathrm{B} 05$, selecting only those $\mathrm{M}$ dwarfs with precise $V$-band magnitudes from CCD photometry. We also collect six high-metallicity examples from JA09. We summarize this calibration sample of M dwarfs in the first 19 lines of Table 1. 
We compute the metallicity predicted for this calibration sample from both the B05 and JA09 relations and compare it to the observed values. Note that the B05 relation was initially based on a calibration sample that included $\mathbf{M}$ dwarfs in binary or multiple systems with FGK primaries (some with $V$-band magnitudes deduced from photographic plates) as well as low-metallicity $\mathrm{M}$ dwarfs with metallicity inferred from spectroscopy after fixing $T_{\text {eff }}$ and $\log g$ with photometric data. Meanwhile, the JA09 calibration sample included only the six metal-rich M dwarfs in binary or multiple systems with FGK primaries listed in rows 14 through 19 in Table 1. For those reasons, we believe that the $19 \mathrm{M}$ dwarfs with metallicities inferred from high-resolution spectroscopy of FGK primaries in Table 1 is the largest and most reliable set of M dwarf metallicities from which to verify previous calibrations. We apply both the B05 and JA09 relations to this sample and compute the residual between each model and observation. We plot the distribution of residuals for both models in Fig. 1, and we find that the B05 relation systematically underestimates M dwarf metallicity and that the JA09 relation systematically overestimates $\mathrm{M}$ dwarf metallicity.

\subsection{A physically-motivated empirical model of $M$ dwarf metallicity}

As discussed in Sect. 2.1 and Fig. 1, the models of B05 and JA09 have non-negligible residuals when applied to the calibration sample in Table 1. Still, there is a correlation between the metallicity of an $\mathrm{M}$ dwarf and its distance in the $\left(V-K_{\mathrm{s}}\right)-M_{K \mathrm{~s}}$ plane from the field M dwarf MS. We attempted to improve the JA09 model by reassessing both the zero point of the model and the direction from the M dwarf MS in the $\left(V-K_{\mathrm{s}}\right)-M_{K \mathrm{~s}}$ plane best correlated with metallicity.

Recall that JA09 set the mean metallicity of the Solar Neighborhood $M$ dwarf sample equal to the mean metallicity of a volume-limited sample of G0-K2 stars $\left(4.0<M_{V}<6.5\right)$ from the SPOCS catalog of Valenti \& Fischer (2005). The SPOCS sample of JA09 was based on a catalog of stars selected to have absorption lines deep enough to enable highprecision radial velocity detection of exoplanets. As a result, the SPOCS sample is biased against metal-poor stars and therefore potentially unsuitable for the determination of the average metallicity in the Solar Neighborhood (as noted in Valenti $\&$ Fischer 2005). Alternatively, the Geneva-Copenhagen Survey (GCS - Nordström et al. 2004; Holmberg et al. 2007, 2009) of Solar Neighborhood F and G dwarfs is magnitude-complete, kinematically-unbiased, and free of the line depth bias inherent in the SPOCS catalog. Though the GCS metallicity estimates are based on Strömgren $u v b y \beta$ photometry and not high-resolution spectroscopy, the precision of the GCS metallicities are sufficient when combined with the reduced bias of the sample to provide a better estimate of the mean Solar Neighborhood metallicity than the SPOCS sample.

In addition, the $U V W$ kinematics of a volume-limited sample of $\mathrm{M}$ dwarfs is not necessarily equivalent to the kinematics of a volume-limited sample of FGK dwarfs. Since the mean of a sample is sensitive to outliers, and because Sun-like stars with outlier kinematics are also likely to be outliers in metallicity, a kinematic-match is important to determine the mean metallicity of Solar Neighborhood M dwarfs in this way. To address this point, we use the $\mathrm{M}$ dwarf $U V W$ distribution described by Hawley et al. (1996) to create a volume-limited and kinematically-matched sample of $F$ and $G$ dwarfs from Holmberg et al. (2009) from which we infer the average metallicity of the Solar Neighborhood M dwarf population. In Fig. 2

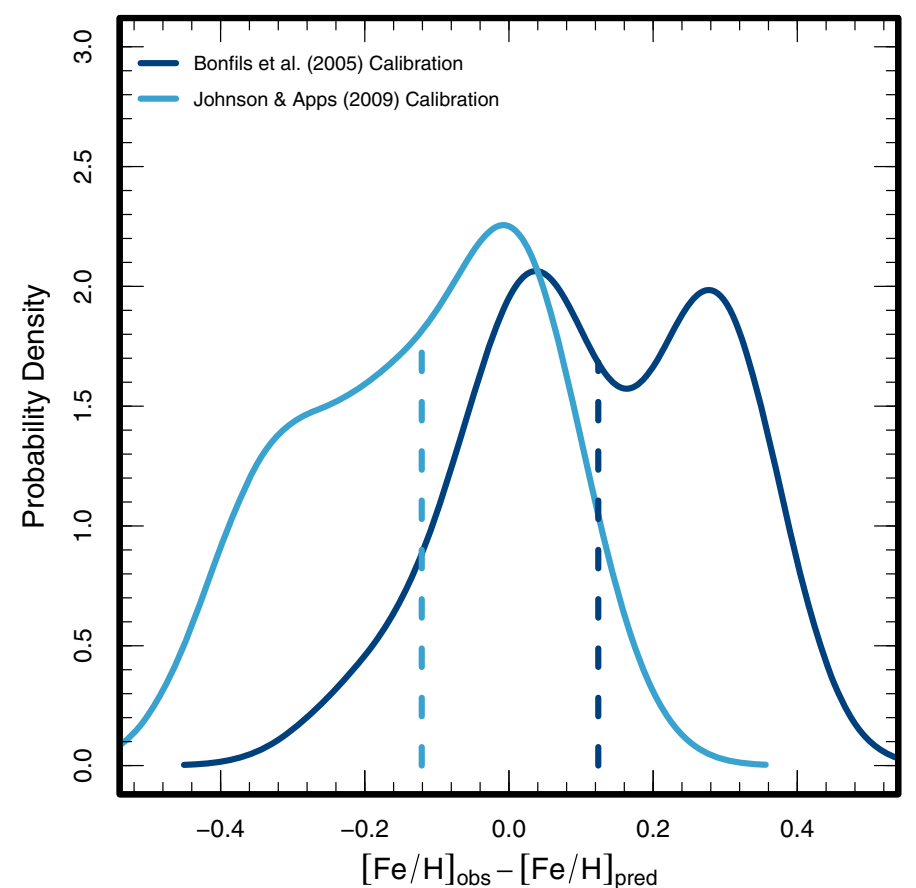

Fig. 1. Optimally-smoothed residual distributions for Bonfils et al. (2005a) - B05 hereafter - and Johnson \& Apps (2009) - JA09 hereafter. In both cases the vertical dashed line indicates the mean of the distribution. The mean value of the $\mathrm{B} 05$ residuals is 0.12 with standard deviation 0.16 , while the mean value of the JA09 residuals is -0.12 with standard deviation 0.12. Note that the B05 distribution has a heavy tail at large positive values (indicating systematically low $[\mathrm{Fe} / \mathrm{H}]$ estimates) and the JA09 distribution has a heavy tail at large negative values (indicating systematically high $[\mathrm{Fe} / \mathrm{H}]$ estimates).

we superimpose the $U V W$ velocity-space distribution of local M dwarfs derived by Hawley et al. (1996) on top of the $U V W$ velocity-space distribution of F and G stars from the GCS with parallax-based distance estimates that place them within $20 \mathrm{pc}$ of the Sun. We bootstrap resample from the subset of GCS stars within $20 \mathrm{pc}$ and with kinematics consistent with the M dwarf velocity ellipsoid as defined in Hawley et al. (1996). We ensure that $68 \%$ of the GCS stars in each bootstrap sample have $U V W$ velocities that place them within the one-sigma contour of Hawley et al. (1996) and that the rest of each bootstrap sample lies within the two-sigma contour. In the end, we find that a volume-limited and kinematically-matched sample of $F$ and G dwarfs from the GCS survey has a mean metallicity of $[\mathrm{Fe} / \mathrm{H}]=-0.14 \pm 0.06$. We obtain a similar result with a sample of GCS stars volume-limited in the same way as the volumelimited SPOCS sample of JA09, for which we find a mean Solar Neighborhood metallicity of $[\mathrm{Fe} / \mathrm{H}]=-0.15 \pm 0.02$. In this case, the superior statistics of the larger volume-limited sample is enough to formally achieve a higher precision than the volume-limited and kinematically-matched sample, though the volume-limited sample is subject to a greater degree of possible systematic error. For that reason, we regard the volume-limited and kinematically-matched result as likely more reliable.

The mean metallicity of our volume-limited and kinematically-matched GCS sample suggests that the field M dwarf MS defined by JA09 is an isometallicity contour with $[\mathrm{Fe} / \mathrm{H}] \approx-0.14$. Note that if the isometallicity contour corresponded to $[\mathrm{Fe} / \mathrm{H}]=-0.05$ as in JA09, five stars from Table 1 with $[\mathrm{Fe} / \mathrm{H}]<-0.05$ would be to the right of the isometallicity contour indicating $[\mathrm{Fe} / \mathrm{H}]>-0.05$. Alternatively, if we assume 

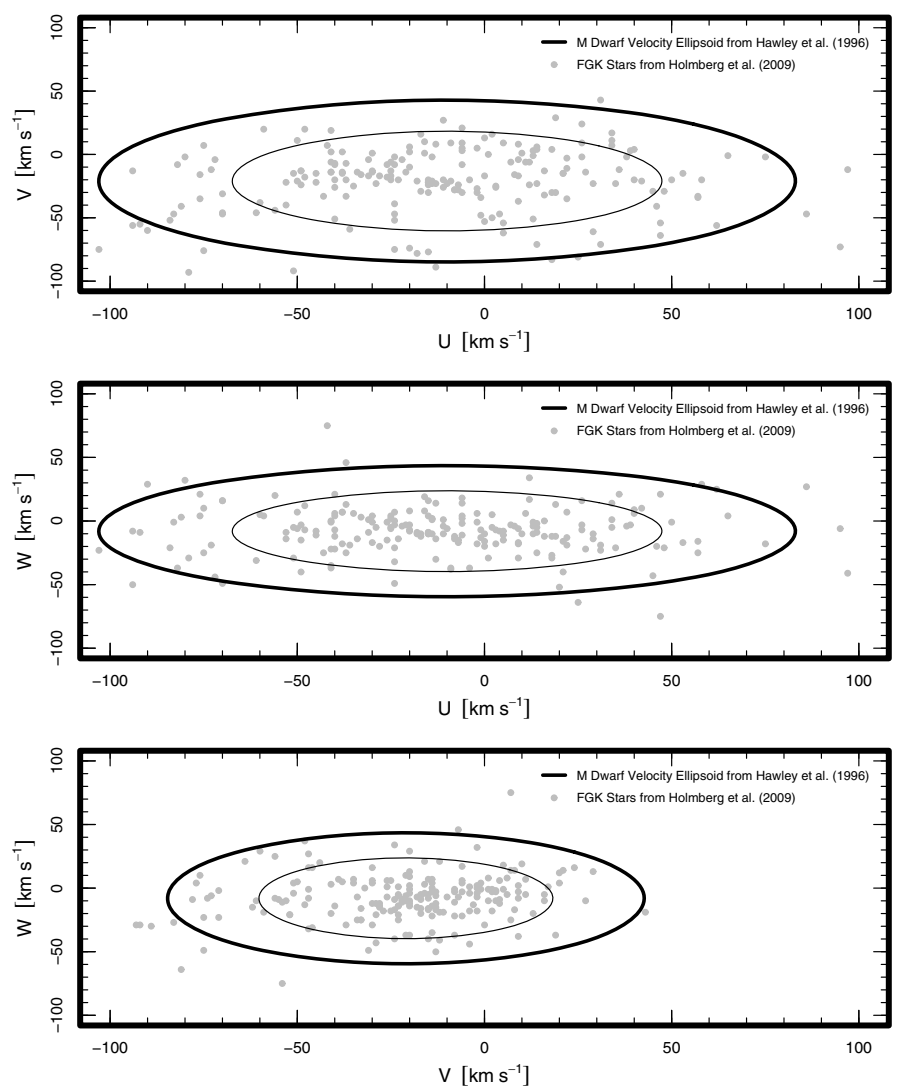

Fig. 2. Velocity ellipsoids inferred for a volume-limited sample of early M dwarfs from Hawley et al. (1996) superimposed on the $U V W$ velocity distribution of a volume-limited sample $(d<20 \mathrm{pc})$ of Sun-like stars from the Geneva-Copenhagen Survey (gray points - Holmberg et al. 2009). The light curve denotes the one-sigma region while the heavy curve denotes the two-sigma region. Bootstrap resampling of the Holmberg et al. (2009) sample with the constraint that $68 \%$ of each bootstrap sample lies within the one-sigma contour and that the other $32 \%$ lies within the two-sigma contour produces a volume-limited and kinematically-matched population metallicity of $[\mathrm{Fe} / \mathrm{H}]=-0.14 \pm 0.06$.

that the isometallicity contour corresponds to $[\mathrm{Fe} / \mathrm{H}] \approx-0.14$, then only two of the 19 stars are on the wrong side of contour.

We now determine which direction in the $\left(V-K_{\mathrm{s}}\right)-M_{K \mathrm{~s}}$ plane an isochrone moves as a function of metallicity. In Fig. 3 we plot the M dwarfs with securely determined metallicity from Table 1, along with the M dwarf MS from JA09 and two different isochrones from Baraffe et al. (1998). We use the transformation of Carpenter (2001) to transform the $K_{\mathrm{CIT}}$ given in Baraffe et al. (1998) into $K_{\mathrm{s}}$. The left-most isochrone corresponds to a population with $[\mathrm{Fe} / \mathrm{H}]=-0.5$ and $Y=0.25$ while the rightmost isochrone corresponds to a population with $[\mathrm{Fe} / \mathrm{H}]=0$ and $Y=0.275$. Both isochrones use mixing-length parameter $l=1$ for a 5 Gyr population (there is no detectable evolution in $\left(V-K_{\mathrm{s}}\right)-M_{K \mathrm{~s}} \mathrm{CMD}$ after $\left.3 \mathrm{Gyr}\right)$. The horizontal lines connect points of constant mass. With all other parameters constant, metallicity should best correlate with horizontal shifts in the $\left(V-K_{\mathrm{s}}\right)-M_{K \mathrm{~s}}$ plane. For that reason, we compute the distance from the M dwarf MS in the horizontal direction for each M dwarf with secure metallicity from Table 1 . We then fit a linear model using this distance as a predictor with $[\mathrm{Fe} / \mathrm{H}]$ as the response. We find that

$[\mathrm{Fe} / \mathrm{H}]=0.79 \Delta\left(V-K_{\mathrm{S}}\right)-0.17$
$\Delta\left(V-K_{\mathrm{s}}\right) \equiv\left(V-K_{\mathrm{s}}\right)_{\mathrm{obs}}-\left(V-K_{\mathrm{s}}\right)_{\text {iso }}$

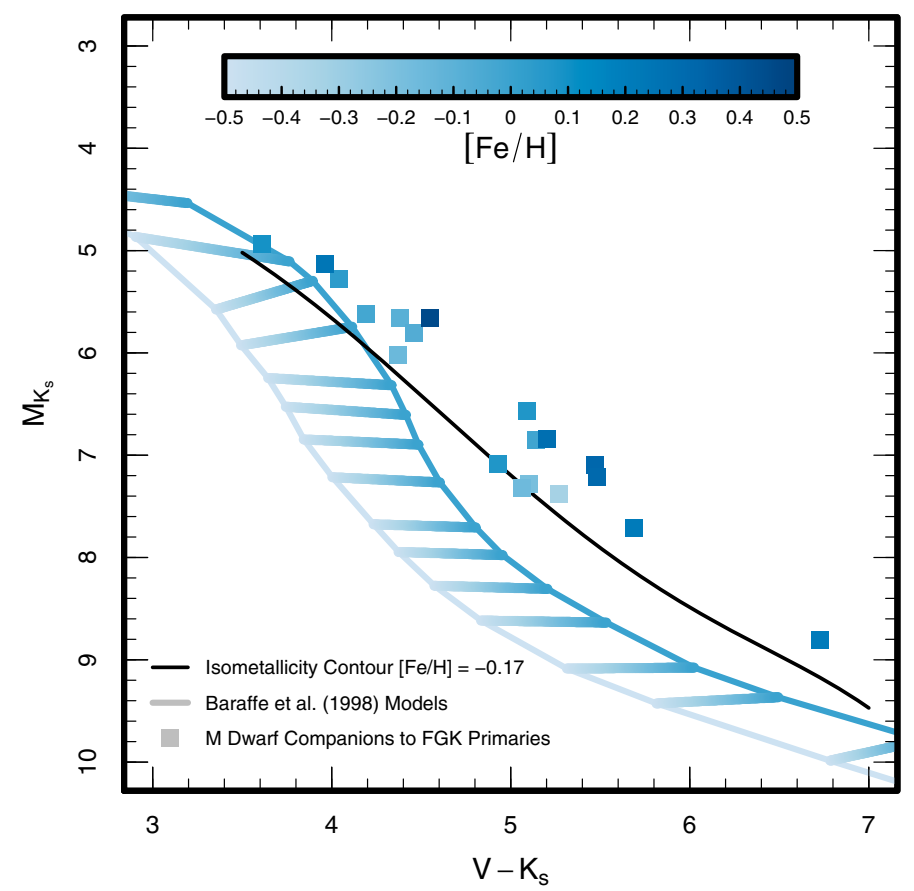

Fig. 3. Position of $M$ dwarfs with secure metallicities from Table 1 (blue points) in the $\left(V-K_{\mathrm{s}}\right)-M_{K \mathrm{~s}} \mathrm{CMD}$ in relation to the field M dwarf MS from JA09 (black line) and the theoretical isochrones of Baraffe et al. (1998) (blue lines). The color of the isochrone line gives its metallicity: $[\mathrm{Fe} / \mathrm{H}]=-0.5$ and $Y=0.25$ on the left and $[\mathrm{Fe} / \mathrm{H}]=0$ and $Y=0.275$ on the right. Both isochrones assume mixing-length parameter $l=1$ for a 5 Gyr population, as there is no detectable evolution in the $(V-$ $\left.K_{\mathrm{s}}\right)-M_{K \mathrm{~s}} \mathrm{CMD}$ after about $3 \mathrm{Gyr}$. The horizontal lines connect points of constant mass. The models indicate that differences in metallicity should best correlate with horizontal shifts in the $\left(V-K_{\mathrm{s}}\right)-M_{K \mathrm{~s}}$ plane.

is the optimal model. In this case, $M_{K \mathrm{~s}}$ as function of $V-K_{\mathrm{s}}$ is given by the fifth-order polynomial with coefficients in increasing order $(-9.58933,17.3952,-8.88365,2.22598,0.258854$, $0.0113399)$ from JA09. To aid in the calculation of $\left(V-K_{\mathrm{s}}\right)_{\text {iso }}$, we give the same curve with $V-K_{\mathrm{s}}$ as a function $M_{K \mathrm{~s}}$ : it is a fifthorder polynomial with coefficients in increasing order (51.1413, $-39.3756,12.2862,-1.83916,0.134266,-0.00382023)$.

We use two of the model selection criteria given in Hocking (1976) to evaluate all three models. First, we compute the residual mean square (rms), defined as

$\mathrm{rms}_{p}=\frac{\mathrm{SSE}_{p}}{n-p}$

where $n$ is the number of data points, $p$ is the number of predictors in the model, and $\mathrm{SSE}_{p}$ is the residual sum of squares for a $p$-term model. In general, models with smaller values of $\mathrm{rms}_{p}$ are best-suited to prediction. For our model, we find that $\mathrm{rms}_{p}=0.02$; for the model of JA09 the value is $\mathrm{rms}_{p}=0.04$ while for the model of B05 the value is $\mathrm{rms}_{p}=0.05$. Next, we compute the adjusted square of the multiple correlation coefficient $R_{a p}^{2}$, which is widely used to judge the fit of a model. A value of $R_{a p}^{2}=1$ indicates that a model explains all of the variance in a sample, while $R_{a p}^{2}=0$ indicates that the model explains none of the variance. $R_{a p}^{2}$ is defined as

$R_{a p}^{2}=1-(n-1) \frac{\mathrm{rms}_{p}}{\mathrm{SST}}$

$\mathrm{SST} \equiv \sum\left(y_{i}-\bar{y}\right)^{2}$ 

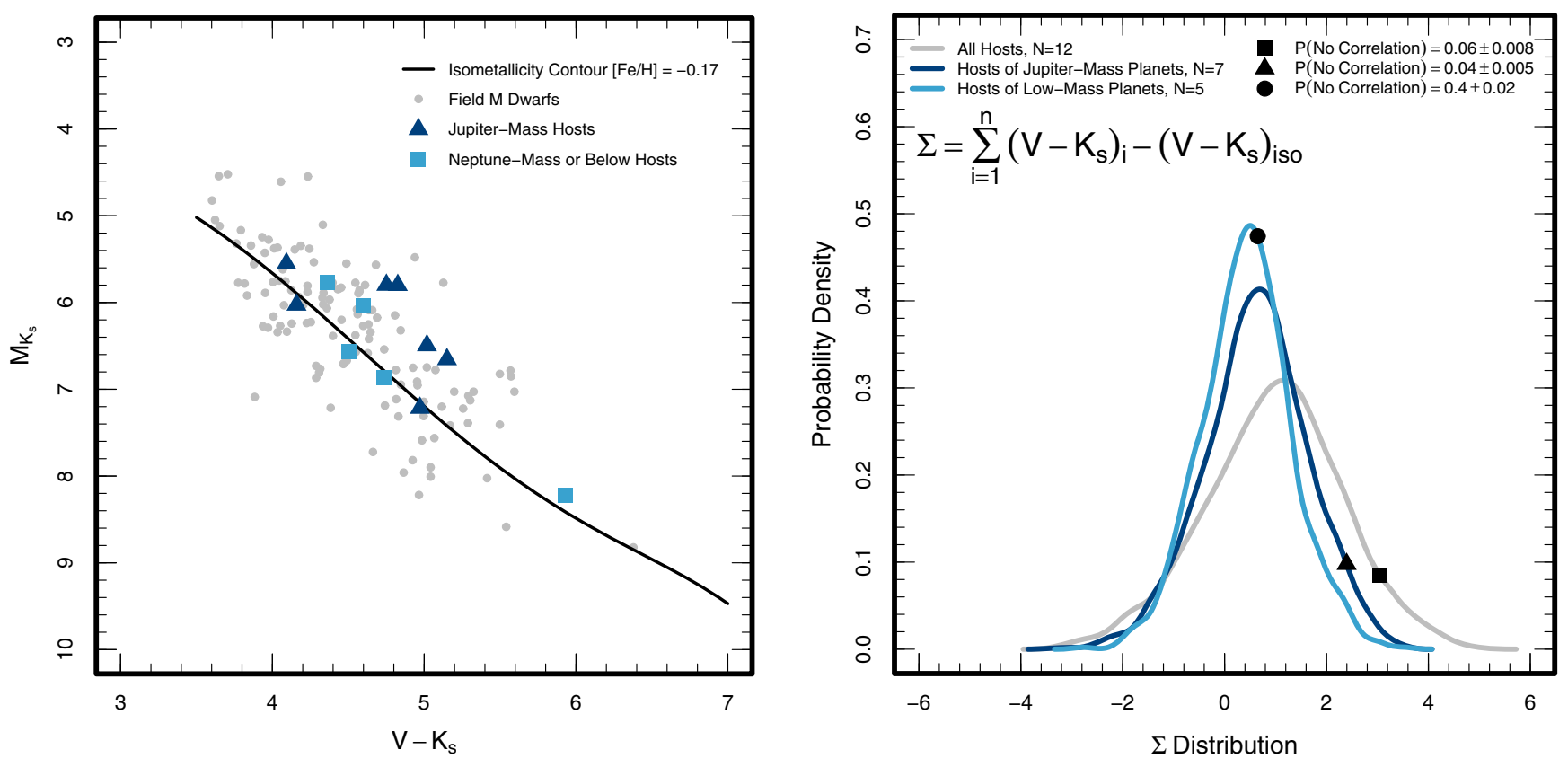

Fig. 4. Left: position of M dwarfs known to host Jupiter-mass planets (dark blue triangles) and Neptune-mass (or below) planets (blue squares) in relation to a control sample of field M dwarfs (gray points) and the field M dwarf MS from JA09 (black line). Again, like the high-metallicity $\mathrm{M}$ dwarfs, the M dwarfs that host planets are concentrated to the right of the field M dwarf MS. Right: distribution of cumulative sample distances from the field M dwarf MS of JA09, which we assume to be a $[\mathrm{Fe} / \mathrm{H}] \approx-0.17$ isometallicity contour in the $\left(V-K_{\mathrm{s}}\right)-M_{K \mathrm{~s}} \mathrm{CMD}$. Points to the right of the field M dwarf MS add their distance to the sum, while points left of MS subtract their distance from the sum. We generate each distribution with a Monte Carlo simulation. First, we randomly select from the field $\mathrm{M}$ dwarf sample a number of stars equal to the number of $\mathrm{M}$ dwarfs known to host planets of a certain type. We then compute the cumulative horizontal distance of that random subsample from the field M dwarf MS. We repeat this process 1000 times to generate the distribution of sample cumulative horizontal distances from the field M dwarf MS given no correlation between the presence of an exoplanet and the location of its host in the $\left(V-K_{\mathrm{s}}\right)-M_{K \mathrm{~s}} \mathrm{CMD}$. In all cases, we confirm the findings of JA09 that the M dwarfs that host exoplanets are preferentially to the right of the field M dwarf MS. In particular, we find that the probability $p$ that there is no correlation between the location of an $\mathrm{M}$ dwarf in the $\left(V-K_{\mathrm{s}}\right)-M_{K \mathrm{~s}} \mathrm{CMD}$ and its status as an exoplanet host is $p=0.06 \pm 0.008$. For the subsample that hosts Jupiter-mass planets, we find that the probability is $p=0.04 \pm 0.005$. More interestingly, we find that the probability that there is no correlation between the location in the $\left(V-K_{\mathrm{s}}\right)-M_{K \mathrm{~s}} \mathrm{CMD}$ and an M dwarf's status as the host of a Neptune-mass (or below) exoplanet is $p=0.40 \pm 0.02$. If $\mathrm{M}$ dwarfs to the right of the field $\mathrm{M}$ dwarf MS are metal-rich as suggested by the Baraffe et al. (1998) models and argued by JA09, then this observation may be evidence for an increased incidence of low-mass planets around metal-rich low-mass stars, a trend which is not observed in FGK stars.

where $y_{i}$ and $\bar{y}$ are the sample and its mean, respectively. For our model, we find that $R_{a p}^{2}=0.49$; for the model of JA09 the value is $R_{a p}^{2}=0.059$ while for the model of B05 the value is $R_{a p}^{2}<0.05$. We note that our model explains almost an order of magnitude more of the variance in the calibration sample than either model presented in B05 or JA09.

Differing $T_{\text {eff }}$ scales have been well-noted as a source of metallicity discrepancies in metallicity studies of the Solar Neighborhood (e.g. Holmberg et al. 2007). The differing $T_{\text {eff }}$ calibrations between the GCS and other surveys will not affect our results, as our calibration (including the metallicity of the $\mathrm{M}$ dwarf MS) is based on the horizontal distance in the $\left(V-K_{\mathrm{S}}\right)-$ $M_{K \mathrm{~s}}$ CMD from the mean M dwarf MS of JA09 of M dwarfs with metallicities known from high-resolution spectroscopy of FGK primaries. We only used the GCS Strömgren-based metallicities to establish the fact that the mean Solar Neighborhood metallicity is closer to $[\mathrm{Fe} / \mathrm{H}] \approx-0.15$ than it is to $[\mathrm{Fe} / \mathrm{H}] \approx$ -0.05 as argued by JA09. For that reason, our calibration is based purely on high-resolution spectroscopy of FGK primaries as well as the trigonometric parallaxes and apparent $V$ - and $K_{\mathrm{s}}$-band magnitudes of their $\mathrm{M}$ dwarf companions.

The $\mathrm{M}$ dwarfs in binary systems with FGK primaries that we use to fix our calibration are not a volumelimited or kinematically-matched sample. The volume-limit and kinematic-match were only necessary to verify the fact that the mean metallicity of the M dwarf population in the Solar Neighborhood is a well-defined quantity. That verification is a necessary precondition that must be established before any JA09 style calibration using distance from the field $\mathrm{M}$ dwarf MS in the $\left(V-K_{\mathrm{s}}\right)-M_{K \mathrm{~s}} \mathrm{CMD}$ can even be considered. Once the points along the field $\mathrm{M}$ dwarf $\mathrm{MS}$ in the $\left(V-K_{\mathrm{S}}\right)-M_{K \mathrm{~s}} \mathrm{CMD}$ are fixed to the mean metallicity of the Solar Neighborhood, the metallicity of an $\mathrm{M}$ dwarf with given $V-K_{\mathrm{s}}$ color and absolute magnitude $M_{K \mathrm{~s}}$ along the curve is specified regardless of its position or velocity. Indeed, when we build our calibration using only the metallicities of $\mathrm{M}$ dwarfs in binaries with FGK primaries, their $V$ - and $K_{\mathrm{s}}$-band magnitudes, and trigonometric parallaxes, we find that the mean metallicity of the Solar Neighborhood M dwarf MS based on the calibration sample $([\mathrm{Fe} / \mathrm{H}]=-0.17 \pm 0.07)$ is statistically indistinguishable from the mean metallicity inferred from the volume-limited and kinematically-matched sample $([\mathrm{Fe} / \mathrm{H}]=-0.14 \pm 0.06)$.

\subsection{The metallicity of $M$ dwarfs that host planets}

We plot the location of M dwarfs that host planets in the lefthand panel of Fig. 4. The M dwarf models of Baraffe et al. (1998) suggest that horizontal distance in the $\left(V-K_{\mathrm{s}}\right)-M_{K \mathrm{~s}} \mathrm{CMD}$ best correlates with metallicity. To non-parametrically determine the degree to which planet-hosting M dwarfs are preferentially 
found to the right of the M dwarf MS, we need to quantify the likelihood that the cumulative horizontal distance from the isometallicity contour of a randomly selected sample of field $\mathrm{M}$ dwarfs can be as large as that observed in the sample of M dwarfs that host planets simply by chance.

To address this issue, we create a control sample of field M dwarfs selected from the Hipparcos (van Leeuwen 2007) and Yale Parallax Catalogs (van Altena et al. 1995). We include in the control sample those $M$ dwarfs from the Hipparcos cata$\log$ that have parallaxes $\pi>100$ mas precise to better than $5 \%$ and those $\mathrm{M}$ dwarfs from the Yale catalog that have parallaxes $\pi>100$ mas. We use $V$-band photometry from each cata$\log$ and we obtain $K_{\mathrm{s}}$ photometry for both samples from the 2MASS database (Skrutskie et al. 2006). For a sample of size $n$ we can compute the statistic $\Sigma$ :

$\Sigma=\sum_{i=1}^{n}\left(V-K_{\mathrm{s}}\right)_{i}-\left(V-K_{\mathrm{S}}\right)_{\text {iso }}$.

To characterize the likelihood that an observed value of $\Sigma$ for a subsample with size $m$ of $\mathrm{M}$ dwarfs that host planets can be produced by chance, we use a Monte Carlo simulation. We randomly select a sample of size $m$ from the $127 \mathrm{M}$ dwarfs in the control sample, compute $\Sigma$ for that sample, save the result, and repeat the calculation 1000 times. In that way, we can determine the distribution of $\Sigma$ expected under the null hypothesis that $\mathrm{M}$ dwarfs that host planets are distributed in the $\left(V-K_{\mathrm{s}}\right)-M_{K \mathrm{~s}} \mathrm{CMD}$ in the same way as field M dwarfs. We consider three sub-samples: (1) all planets hosts; (2) hosts of Jupiter-mass planets; and (3) hosts of Neptune-mass (and below) planets. We find that in case (1) $\Sigma=3.43$ indicating only a $p=0.06 \pm 0.008$ probability that the cumulative distance of the sample from the isometallicity contour occurred by chance. In case (2) we find $\Sigma=2.39 \Rightarrow p=0.04 \pm 0.005$ and in case (3) we find $\Sigma=1.04 \Rightarrow p=0.40 \pm 0.02$. We summarize this calculation in the right-hand panel of Fig. 4.

\subsection{Discussion}

The apparent position of planet-hosting $\mathrm{M}$ dwarfs in the region of the $\left(V-K_{\mathrm{s}}\right)-M_{K \mathrm{~s}} \mathrm{CMD}$ associated with known high-metallicity $\mathrm{M}$ dwarfs tentatively suggests that metal-rich M dwarfs are more likely to host Jupiter-mass and possibly Neptune-mass planets as well. If this correlation is confirmed in the future, it can be understood as a natural consequence of the core-accretion model of planet formation (e.g. Laughlin et al. 2004; Ida \& Lin 2004). Indeed, a more metal-rich protoplanetary disk will almost certainly have a higher surface density of solids, and that increased surface density enables the rapid formation of the several Earth-mass cores necessary to accrete gas from the protoplanetary disk before the gaseous disk is dissipated. Moreover, it would be especially interesting if the tentatively suggested correlation extends to the hosts of Neptune-mass planets, as current evidence seems to suggest that the probability that an FGK star hosts a Neptune-mass planet is not a strong function of metallicity (e.g. Udry et al. 2006; Sousa et al. 2008; Bouchy et al. 2009).

If the tentatively suggested correlation between the presence of planets and the metallicity of their host M dwarfs is eventually confirmed, it might indicate a lower-limit on the amount of solid material necessary to form planets. To see why, recall that the mass of a protoplanetary disk scales roughly as $M_{\text {disk }} \propto M_{*}$ and that the fraction of solid material in a disk $f_{\text {solid }}$ scales roughly as $Z_{*}$ where $Z_{*}$ is the metal content of the host star. The total amount of solid material in a protoplanetary disk will then scale like $M_{\text {solid }} \propto f_{\text {solid }} M_{\text {disk }} \propto Z_{*} M_{*}$. Minimum-mass Solar Nebula models (MMSN - Hayashi 1981) and observations of T Tauri disks in star-forming regions suggest that protoplanetary disks around young Solar-type stars are about $1 \%$ the mass of their host stars, albeit with significant scatter (e.g. Hartmann et al. 1998). Combined with the fact that the metal content of the Sun is $Z_{\odot}=0.0176$ by mass, the total solid mass in the MMSN was about $M_{\text {solid }} \approx 60 M_{\oplus}$. This is a lower-limit, as more careful calculations suggest that the protoplanetary disk around the Sun had $M_{\text {solid }} \sim 100 M_{\oplus}$ (e.g. Lissauer 1993). In either case, this is a factor of a few to ten greater than the $10 M_{\oplus}$ of material necessary to form the core of a gas or ice giant planet in the core-accretion model of planet formation. In the case of a Solarmetallicity mid-M dwarf with $M_{*}=0.3 M_{\odot}$, the total amount of solid material in the disk is $70 \%$ less, about $M_{\text {solid }} \approx 20 M_{\oplus}$. This is factor of order unity to a few times the mass necessary to form the core of a gas or ice giant. Since planet formation likely does not lock-up the entire solid component of a protoplanetary disk in planets, reducing the total mass of solids in the disk - either by reducing the metallicity or mass - will also reduce the chances of forming a $10 M_{\oplus}$ core (and therefore a gas or ice giant) before the parent protoplanetary disk is dissipated.

The confirmation of the hint of a correlation between the presence of low-mass planets and M dwarf metallicity could be evidence of this threshold solid mass necessary to form Neptune-mass planets. Around FGK stars, the same threshold solid mass suggests that a correlation between the presence of low-mass planets and host star metallicity might occur at onethird Solar metallicity, or $[\mathrm{Fe} / \mathrm{H}]=-0.5$. This is just below the typical metallicity of stars observed at high radial velocity precision with HARPS (Sousa et al. 2008). This expected correlation might be verified as larger samples of low-metallicity stars are surveyed at high radial velocity precision or by transit surveys of nearby low-metallicity open clusters (e.g. NGC 752 or IC 4756).

The hint of a correlation between the presence of Neptunemass (and below) planets and M dwarf metallicity tentatively suggests that searches for low-mass planets around M dwarfs like the MEarth Project (Nutzman \& Charbonneau 2008) could improve their yield by shading their target list toward $\mathrm{M}$ dwarfs that have red $V-K_{\mathrm{S}}$ colors at constant $K_{\mathrm{s}}$-band absolute magnitude $M_{K \mathrm{~s}}$. Note that since the absolute magnitude $M_{K \mathrm{~s}}$ of an M dwarf depends only on the logarithm of its often poorlyknown distance, while $V-K_{\mathrm{s}}$ depends linearly on its often poorly-known $V$-band magnitude, the collection of high-quality CCD-based $V$-band magnitudes for $M$ dwarfs in the Solar Neighborhood could be the first step towards maximizing the yield of planets around $\mathrm{M}$ dwarfs.

\section{Conclusion}

We showed that previous empirical photometric calibrations of M dwarf metallicity systematically underestimate or overestimate metallicity at the extremes of their range. We derived a physically-motivated model that explains an order of magnitude more of the variance in the calibration sample than either the Bonfils et al. (2005a) or Johnson \& Apps (2009) models. We used the correlation underlying our model to non-parametrically show that the probability $p$ that there is no relationship between position of an $\mathrm{M}$ dwarf in the $\left(V-K_{\mathrm{s}}\right)-M_{K \mathrm{~s}} \mathrm{CMD}$ and the presence or absence of planets is $p=0.06 \pm 0.008$. For the subsample of $\mathrm{M}$ dwarfs that host Jupiter-mass planets, the probability that there is no correlation is $p=0.04 \pm 0.005$. Meanwhile, for the subsample of $\mathrm{M}$ dwarfs that host Neptune-mass (or below) 
planets, we find that the probability that there is no correlation is $p=0.40 \pm 0.02$. Since the models of Baraffe et al. (1998) suggest that the position of an $\mathrm{M}$ dwarf in the $\left(V-K_{\mathrm{s}}\right)-M_{K \mathrm{~s}} \mathrm{CMD}$ is a qualitative indicator of metallicity, this observation tentatively suggests that metal-rich $\mathrm{M}$ dwarfs are more likely to host planets and hints that the correlation may extend to low-mass planets as well. If this correlation is confirmed in the future, it will be in contrast to planetary systems around FGK stars, in which there appears to be only a weak connection between metallicity and the presence of Neptune-mass planets.

Acknowledgements. We thank Connie Rockosi for useful comments and conversation and the anonymous referee for many insightful suggestions that improved this paper significantly. This research has made use of NASA's Astrophysics Data System Bibliographic Services, the Exoplanet Orbit Database and the Exoplanet Data Explorer at exoplanets.org, and the NASA/IPAC Infrared Science Archive, which is operated by the Jet Propulsion Laboratory, California Institute of Technology, under contract with the National Aeronautics and Space Administration. This material is based upon work supported under a National Science Foundation Graduate Research Fellowship.

\section{References}

Apps, K., Clubb, K. I., Fischer, D. A., et al. 2010, PASP, 122, 156 Bailey, J., Butler, R. P., Tinney, C. G., et al. 2009, ApJ, 690, 743

Baraffe, I., Chabrier, G., Allard, F., \& Hauschildt, P. H. 1998, A\&A, 337, 403 Bonfils, X., Delfosse, X., Udry, S., et al. 2005a, A\&A, 442, 635

Bonfils, X., Forveille, T., Delfosse, X., et al. 2005b, A\&A, 443, L15

Bonfils, X., Mayor, M., Delfosse, X., et al. 2007, A\&A, 474, 293

Bouchy, F., Mayor, M., Lovis, C., et al. 2009, A\&A, 496, 527

Butler, R. P., Johnson, J. A., Marcy, G. W., et al. 2006, PASP, 118, 1685

Carpenter, J. M. 2001, AJ, 121, 2851

Charbonneau, D., Berta, Z. K., Irwin, J., et al. 2009, Nature, 462, 891
Egret, D., Didelon, P., McLean, B. J., Russell, J. L., \& Turon, C. 1992, A\&A, 258, 217

Fischer, D. A., \& Valenti, J. 2005, ApJ, 622, 1102

Forveille, T., Bonfils, X., Delfosse, X., et al. 2009, A\&A, 493, 645

Gliese, W., \& Jahreiß, H. 1991, Preliminary Version of the Third Catalogue of Nearby Stars, Tech. Rep.

Gustafsson, B. 1989, ARA\&A, 27, 701

Hawley, S. L., Gizis, J. E., \& Reid, I. N. 1996, AJ, 112, 2799

Hartmann, L., Calvet, N., Gullbring, E., \& D’Alessio, P. 1998, ApJ, 495, 385

Hayashi, C. 1981, Prog. Theor. Phys. Suppl., 70, 35

Hocking, R. R. 1976, Biometrics, 32, 1

Holmberg, J., Nordström, B., \& Andersen, J. 2007, A\&A, 475, 519

Holmberg, J., Nordström, B., \& Andersen, J. 2009, A\&A, 501, 941

Howard, A. W., Johnson, J. A., Marcy, G. W., et al. 2010, ApJ, submitted [arXiv: 1003.3488]

Ida, S., \& Lin, D. N. C. 2004, ApJ, 616, 567

Johnson, J. A., \& Apps, K. 2009, ApJ, 699, 933

Johnson, J. A., Butler, R. P., Marcy, G. W., et al. 2007, ApJ, 670, 833

Johnson, J. A., Howard, A. W., Marcy, G. W., et al. 2010, PASP, 122, 149

Laughlin, G., Bodenheimer, P., \& Adams, F. C. 2004, ApJ, 612, L73

Lissauer, J. J. 1993, ARA\&A, 31, 129

Maness, H. L., Marcy, G. W., Ford, E. B., et al. 2007, PASP, 119, 90

Marcy, G. W., Butler, R. P., Vogt, S. S., Fischer, D., \& Lissauer, J. J. 1998, ApJ, $505, \mathrm{~L} 147$

Mermilliod, J.-C., Mermilliod, M., \& Hauck, B. 1997, A\&AS, 124, 349

Nordström, B., Mayor, M., Andersen, J., et al. 2004, A\&A, 418, 989

Nutzman, P., \& Charbonneau, D. 2008, PASP, 120, 317

Perryman, M. A. C., Lindegren, L., Kovalevsky, J., et al. 1997, A\&A, 323, L49

Santos, N. C., Israelian, G., \& Mayor, M. 2004, A\&A, 415, 1153

Skrutskie, M. F., Cutri, R. M., Stiening, R., et al. 2006, AJ, 131, 1163

Sousa, S. G., Santos, N. C., Mayor, M., et al. 2008, A\&A, 487, 373

Udry, S., Mayor, M., Benz, W., et al. 2006, A\&A, 447, 361

van Altena, W. F., Lee, J. T., \& Hoffleit, E. D. 1995, New Haven, CT: Yale

University Observatory, 4th edn., completely revised and enlarged

Valenti, J. A., \& Fischer, D. A. 2005, ApJS, 159, 141

van Leeuwen, F. 2007, A\&A, 474, 653 